\title{
Problems with evaluating readings of pulse oximeters used at home during the ongoing COVID-19 pandemic
}

\author{
A. Cysewska-Sobusiak ${ }^{*}$ \\ Institute of Electrical Engineering and Electronics, \\ Poznan University of Technology, Piotrowo 3a, 60-965 Poznan
}

Received March 16, 2021; accepted March 29, 2021; published March 31, 2021

\begin{abstract}
The author presents the problems related to pulse oximetry, considering both the current state-of-the-art as well as her own long-standing experience in this field. The COVID-19 pandemic continues and has raised interest in home application of pulse oximeters due to their potential benefit, which may be warning of a drop in blood oxygenation due to lower respiratory capacity. However, it should be emphasized that these devices have limitations, both of a physiological and technical nature, and some problems related to the reliability and scope of the measurements still remain unresolved. There are presented phenomena which limit processing reliability of the quantity to be sensed, i.e., oxygen saturation $\mathrm{SaO}_{2} \%$. There are distinguished specific groups of contributing factors, at an attempt to reveal their effect on measurement uncertainty.
\end{abstract}

One measure of human tissues vitality is a blood oxygen content, which is one of the most requested tests performed on critically ill patients. Arterial blood is of the same saturation throughout the arterial system. The result is expressed as a percentage ratio of oxygenated hemoglobin $\left(\mathrm{HbO}_{2}\right)$ to the total amount of hemoglobin $\left(\mathrm{Hb}+\mathrm{HbO}_{2}\right)$. It is called oxygen saturation $\mathrm{SaO}_{2} \%$. Pulse oximetry is a marvellous example of optically based diagnostic methods which allow noninvasive monitoring of this saturation [1]. Tissue transillumination windows extend from 600 to $1200 \mathrm{~nm}$ [2]. Selective changes in the absorption of detected hemoglobins are shown in Fig. 1.

Measurable biooptical signals induced by peripheral pulse waveforms can be acquired with optoelectronic sensors placed on peripheral body sites. Transilluminated objects have to be optically thin. From the measurement point of view, especially convenient for effective transillumination are hand fingers (Fig. 2), which are widely used as objects in the transmission variants of pulse oximetry. The transmission sensors in modern pulse oximeters usually use two wavelengths (660 nm and 940 $\mathrm{nm}$ ) capable of detecting differences in optical properties of oxygenated and reduced hemoglobins [3]. Hemoglobins absorb different amounts of light at different wavelengths, depending on the level of oxygen they are carrying. The sensor should be equipped with a high-efficient incident light emitter (containing either a single LED or a set of LEDs) and a sensitive output light

\footnotetext{
*E-mail: anna.cysewska@put.poznan.pl
}

detector (containing either a single photodiode or a set of photodiodes).

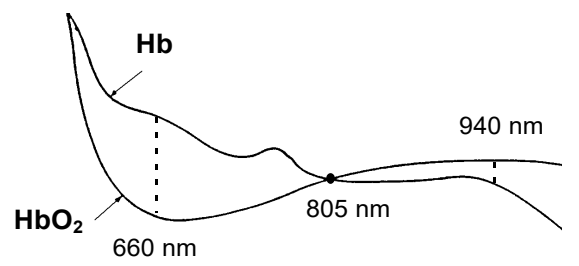

Fig. 1. Illustration of changes in the absorption of both hemoglobins: oxygenated $\mathrm{HbO}_{2}$ and reduced $\mathrm{Hb}$ in relation to the wavelength.

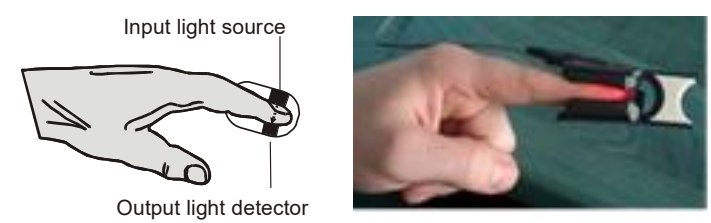

Fig. 2. Noninvasive finger-tip sensor used in a transmission variant of pulse oximetry.

Transmission pulse oximetry smartly joins both the rules of in vivo spectrophotometry and transmission pulse waveforms in monitoring arterial blood oxygen saturation. Continuous monitoring may be performed at rest, during activity or sleep. However, pulse oximeter indications $\mathrm{SpO}_{2} \%$ estimate the true value $\mathrm{SaO}_{2} \%$ with uncertainty. The true value is given as $\mathrm{SaO}_{2} \%=\mathrm{HbO}_{2} \% /\left(\mathrm{Hb} \%+\mathrm{HbO}_{2} \%\right)$ while any numerical indication of a pulse oximeter is: $\mathrm{SpO}_{2} \%=\mathrm{SaO}_{2} \% \pm \Delta \%$.

Early evaluations of blood oxygenation with bloodless techniques based on light-tissue interaction were unacceptable in practice; however, the progress which has been made in this area is enormous. Unique advantages of pulse oximetry have made pulse oximeters recommended standard equipment in intensive care and other critical situations related to impending hypoxemia [4]. Currently COVID-19 is an emerging, rapidly evolving situation when the awful virus causes a respiratory disease in which patients often complain of shortness of breath, fever, cough and fatigue. The anxiety that always accompanies pandemics also has an impact on mental 
health [5]. Social distancing is not a new idea: it saved many lives during the last great pandemic of 1918 [6]. In addition to social distancing, mask wearing and frequent handwashing, technology can also help fight the virus that causes COVID-19, having a calming effect on people. A supportive technical supplement, an easily available device of low-complexity and low-cost, may enable noninvasive home control of arterial blood oxygenation. Because the pandemic still persists, many people become very sensitive to such terms as "oxygen saturation" and "pulse oximeter" in the context of ongoing infection. However, end-users of pulse oximeters are not always aware that these devices have their specific limitations, affecting sensitivity, resolution, and repeatability of processing function, as well as response time and stability of results.

A sensor may be attached to a finger for monitoring beat-to-beat pulsation as well as the shape of a peripheral pulse waveform. The shape of this curve largely depends on the blood supply extent of the area examined.

Some popular smart watches have a pulse oximetry function, but they should not be referred to as reliable $\mathrm{SpO}_{2}$ sensors because they are not intended for medical purposes. They may be useful for recreational purposes, helping to determine how a body can acclimatize to high altitudes.

In clinical conditions, there are used stationary devices, to which the required sensor is connected. A compact pulse oximeter is a small measuring device with an integrated finger sensor. But such a device is able to show a pulse waveform, heart rate and $\mathrm{SpO}_{2} \%$ (Fig. 3).

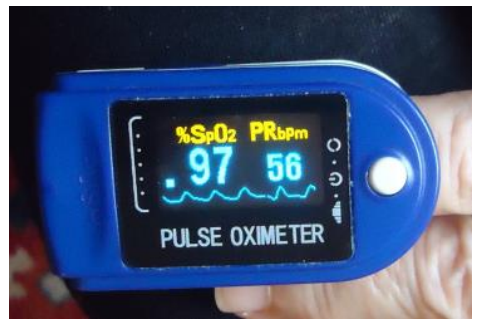

Fig. 3. Example of a reading obtained at home with a compact pulse oximeter.

It is not recommended to use a pulse oximeter to selfdiagnose COVID-19. If someone has the symptoms of an infection or has been in contact with an infected person, a test must be done. Conversely, if someone has been diagnosed with COVID-19, a pulse oximeter can be a helpful tool to monitor their health and tell them if medical attention is needed to reduce the risk of serious health deterioration. On the one hand, many people with COVID-19 have low oxygenation and feel well. On the other hand, some people may feel very sick and have a good level of oxygenation. A low saturation value can therefore be an early warning signal that someone needs medical attention.
Home pulse oximeters may be very useful for patients with COVID-19 who can experience a potentially dangerous drop in oxygen saturation without having obvious breathing problems. Finger clip technology is now accepted. After placing a finger inside the device, within seconds lighting numbers indicate heart rate and $\mathrm{SpO}_{2} \%$ blood oxygen saturation. Healthy adult people will usually get a normal resting heart rate from 60 to 100 beats per minute and a normal oxygen saturation reading from 95 to 98 percent.

Medical intervention is necessary if oxygen saturation levels began to fall. Because oxygen levels can fluctuate, the users should remember that what matters is the trend of changes, not a single reading. The best pulse oximetry threshold for detecting hypoxia is $92 \%$. Everybody should check in with a doctor if the indicated $\mathrm{SpO}_{2} \%$ number falls to around 92 or lower.

Clinical tests compare pulse oximeter saturation readings to arterial blood gas saturation readings for the values between $70 \div 100 \%$. Reading accuracy is highest at saturations of $90 \div 100 \%$, intermediate at $80 \div 90 \%$, and lowest below $80 \%$. If the $\mathrm{SaO}_{2} \%$ is below $70 \%$, the accuracy of $\mathrm{SpO}_{2} \%$ reading will be unknown. From the metrological point of view, a typical uncertainty interval is quite wide. For example, if an $\mathrm{SpO}_{2} \%$ reading is $94 \%$, then true oxygen saturation of arterial blood may be between $90 \div 98 \%$. Pulse oximeters can overestimate $\mathrm{SaO}_{2}$

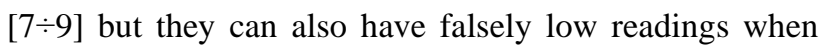
applied, e.g. to cold fingers.

All pulse oximeters have limitations and carry a risk of inaccuracy because multiple factors can affect the reliability of a given device reading. The author has long experience in the field and made a whole set of experiments under laboratory, as well as clinical conditions $[10 \div 13]$. Different intrinsic and extraneous quantities and factors, which can influence the reliability of results, have been shown in Fig. 4.

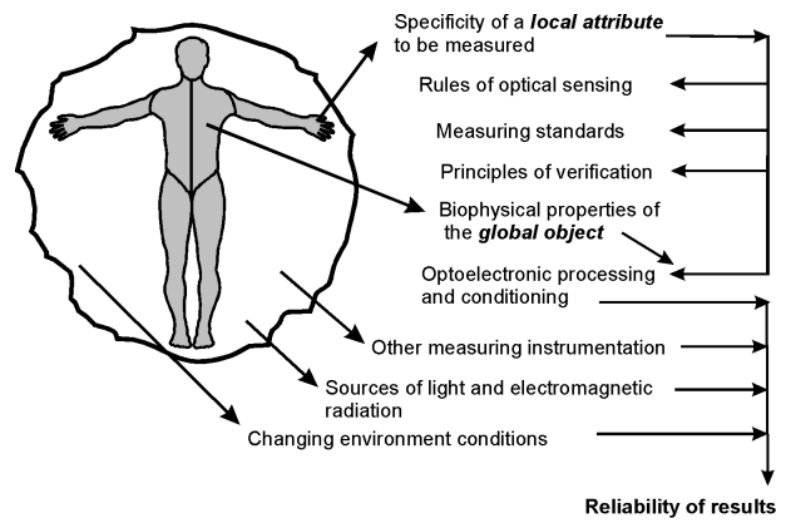

Fig. 4. Quantities and factors that can reduce the reliability of readings in pulse oximetry. 
Intrinsic factors result from a decrease in the size of arterial pulsations caused by: a weak and/or abnormal pulse waveform, abnormal thickness or other properties of the object (hypothermia, cardiac arrest, hypotension, anemia), deep hypoxemia, or from the presence of any internal absorbers, except $\mathrm{HbO}_{2}$ and $\mathrm{Hb}$, which are able to affect transillumination effectiveness and processing reliability (e.g. pathological fractions of dyshemoglobins).

Extraneous factors are connected with forced moves of the object after sensor application and/or forced changes in the object's features. The latter can induce a loss of pulse and artificial changes in pigmentation. The influences of other measuring instrumentation and diagnostic tools as well as environment can also disturb measurements and distort their results.

It is evident that arterial pulsations are necessary to sense the oxygenated hemoglobin content with pulse oximetry. Many difficulties with sensing result from the fact that affecting disturbances often occur at frequencies within the physiological ranges of converted useful signals.

Any rhythmical or irregular changes in the pulsatile component of the distance between the light emitter and detector, and in the pulsatile part of transmitted light intensity can cause noticed instantaneous changes in the peak and bottom values of a pulse component, affecting the processing function.

Multiple factors can limit the reliability of measurement, including causes such as improper structure or finger injury, improper sensor placement, patient movement, presence of intravascular pigments, temperature and ambient light effects, as well as disturbed breathing and arrhythmia.

As was mentioned above, the device works better with warmer fingers than cold fingers. Dark nail polish can affect the accuracy of a reading. Very long nails would make it difficult to insert a finger properly in the clip. A sensor may not be put on the same hand as the blood pressure cuff.

In conclusion of this short presentation, it may be stated that:

- Early noninvasive measurements of $\mathrm{SaO} 2$ were limited to clinical conditions, of special value in anesthesia and intensive care, and in all hospital sites where patients are not able to regulate their own oxygen supply.

- Currently widespread use of pulse oximetry works better on more ambulatory patients with special interest in problems caused by COVID-19, which is an emerging, rapidly evolving situation.
- Decrease in measurement accuracy can manifest three-fold: first, by a loss of ability to detect changes in true $\mathrm{SaO}_{2} \%$, second, by indications $\mathrm{SpO}_{2} \%$ overestimating or underestimating the sensed value, and third, by a lack of stability of results to be obtained in a series of measurements on the same human subject.

- Any user of a pulse oximeter may obtain, at the same true $\mathrm{SaO}_{2} \%$ value, another $\mathrm{SpO}_{2} \%$, value which depends on individual features of the object and always underestimates or overestimates $\mathrm{SaO}_{2} \%$. Inaccurate measurements are caused by incorrect sensor application, as well as interferences of different nature and importance.

- Manufactures should determine in their operation guides the principles as well as warnings under which the $\mathrm{SpO}_{2} \%$ indications might be reliable within a given uncertainty.

- In addition to social distancing, mask wearing and frequent handwashing, as well as technology can help fight the virus that causes COVID-19, having a calming effect on people. Such a supportive technical supplement may be a currently available, lowcomplexity and low-cost pulse oximeter, enabling home, non-invasive control of arterial blood oxygenation.

- In home monitoring, such a simple, compact device with an integrated finger sensor can help alert patients and make them seek specialist help before they arrive at hospital with dangerously low oxygen levels, risking serious complications and death. It seems that it is worth getting this device, which is a kind of useful gadget of the COVID-19 pandemic.

\section{References}

[1] J.W. Severinghaus, Adv. Exp. Med. Biol. 812 (2014).

[2] F.A. Duck, Physical properties of tissue: a comprehensive reference book (San Diego, Academia Press 1990).

[3] J.G. Webster, Design of pulse oximeters (Bristol, IOP Publishing Ltd 1997).

[4] A.A. Alian, K.H Shelley, Anesthesiology 28, 4 (2014).

[5] N. Moukaddam, A. Shah, Psychiatric Times 37, 3 (2020).

[6] S.S. Morse, Proc. Nat. Acad. Sci. 104, 18 (2007).

[7] W.A. Schrading, B. McCafferty, J. Grove, D.B. Page, JACP Open 1 (2020).

[8] K.E.J. Philip, B. Bennet, S. Fuller et al., BMJ Open Resp. Res. 7 (2020).

[9] S. Seifi, A. Khatony, G. Moradi, A. Abdi, F. Najafi, BMC Nursing 17, 15 (2018)

[10] A. Cysewska-Sobusiak, Measurement 25 (1999).

[11] A. Cysewska-Sobusiak, Biocybernetics and Biomedical Engineering 22, 4 (2002).

[12] A. Cysewska-Sobusiak, $3^{\text {rd }}$ Int. Workshop on Robot Motion and Control, Nov. 2002 (2002).

[13] A. Cysewska-Sobusiak, Phot. Lett. Poland 11, 2 (2019). 